REVISTA MATEMÁTICA de la

Universidad Complutense de Madrid

Volumen 6, número 1; 1993.

http://dx.doi.org/10.5209/rev_REMA.1993.v6.n1.17839

\title{
The Tensor Product of Triples as Multilinear Product*
}

Jose L. Freire Nistal AND Miguel A. LoPez LoPEZ

ABSTRACT. In this paper we introduce a notion of multilinear product for triples in Set, which if it is given by a distributive law then coincides with the one given by Bunge. We also demonstrate that the tensor product of two triples, if there exist, is an initial object in a suitable category of multilinear products.

\section{INTRODUCTION}

In "Producto de triples" ([8]) the definition that is given of the product of triples generalizes the notion of distributive law, according to Beck ([2]). The tensor product is studied by $\mathrm{E}$. Manes in various articles ([10], [11], [12]), for triples in the category Set, of sets and maps.

M. Bunge, in ([3]), studies the relationship between composition triple and tensor product of triples, for triples in Set. In this paper, it is given the definition of distributive multilinear law, which is the

* Supported by grant TIC91-0404 of Comisión Interministerial de Ciencia y Tecnologia (Spain).

1991 Mathematics Subject Classification: $18 \mathrm{C15}$.

Editorial Complutense. Madrid, 1993. 
distributive law in which each algebra over the composition triple is also a bialgebra.

The aim of the present paper is to introduce a notion of multilinear product for triples in Set, which if it is given by a distributive law then coincides with the one given by Bunge, and to demonstrate that the tensor product of two triples $\mathbf{T}$ and $\mathbf{S}$, if there exist, is an initial object in the category whose objects are multilinear products $R=(\mathbf{T S})_{\mathbf{r}}$, and whose morphisms $\alpha: \mathbf{R} \rightarrow \mathbf{R}^{\prime}$ are morphisms of triples that make the following diagram commutative

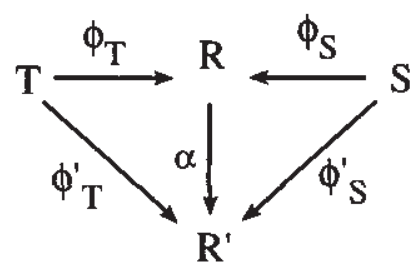

$\phi_{\mathrm{T}}$ and $\phi_{\mathrm{S}}$ being the morphisms of triples asociated to every product ([8]).

\section{PRODUCT OF TRIPLES AND DISTRIBUTIVE LAWS}

1.1 If $\mathbf{T}=\left(T, \eta_{T}, \mu_{T}\right)$ and $S=\left(S, \eta_{s}, \mu_{S}\right)$ are triples in a category $A$, a product $\mathbf{R}=(\mathbf{T S})_{\mathrm{r}}$ is a triple $\mathbf{R}=(\mathbf{R}, \eta, \mathrm{m})$, where $\eta=r \circ\left(\eta_{\mathrm{T}} * \eta_{\mathrm{S}}\right)$ and where the natural tranformation $\mathrm{r}: \mathrm{TS} \Rightarrow \mathrm{R}$ verifies the following axioms:
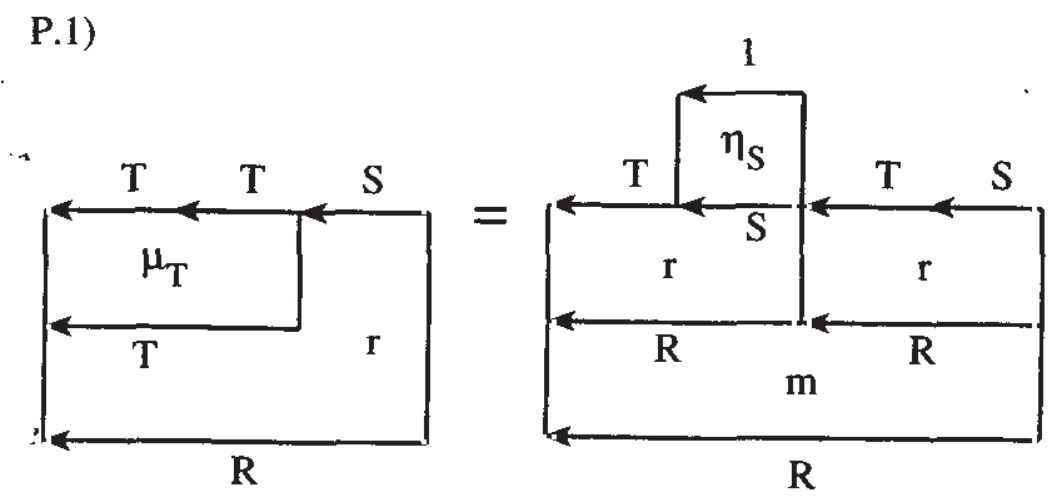

i.e.: $r \circ\left(\mu_{T} * S\right)=m \circ(r * r) \circ\left(T * \eta_{S} * T S\right)$ 
P.2)

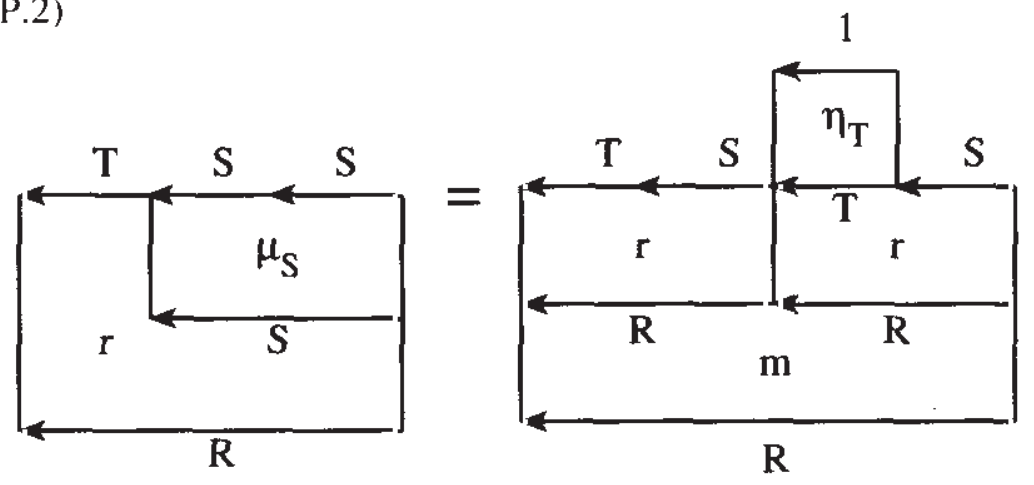

i.e.: $r \circ\left(T \star \mu_{\mathrm{S}}\right)=\mathrm{m} \circ(\mathrm{r} \star \mathrm{r}) \circ\left(\mathrm{TS} \star \eta_{\mathrm{T}} \star S\right)([8], 1.1)$.

1.2 If $R=(\mathbf{T S})_{\mathrm{r}}$ is a product, then $\phi_{\mathrm{T}}:=\mathrm{r} \circ\left(\mathrm{T} * \eta_{\mathrm{S}}\right): \mathbf{T} \Rightarrow \mathbf{R}$ and $\phi_{\mathrm{S}}:=$ $\mathrm{r} \circ\left(\eta_{\mathrm{T}} \star S\right): \mathbf{S} \Rightarrow \mathbf{R}$ are morphisms of triples.

Conversely, if $\phi_{\mathrm{T}}: \mathbf{T} \Rightarrow \mathbf{R}$ and $\phi_{\mathrm{S}}: \mathbf{S} \Rightarrow \mathbf{R}$ are morphisms of triples, with $\mathbf{R}=\left(\mathrm{R}, \eta_{\mathrm{R}}, \mathrm{m}\right)$ then $\mathbf{R}=(\mathrm{TS})_{\mathrm{r}}$ with $\mathrm{r}:=\mathrm{m} \circ\left(\phi_{\mathrm{T} *} \phi_{\mathrm{S}}\right)$ : TS $\Rightarrow \mathrm{R}$.

Moreover, if $\mathbf{R}=(\mathbf{T S})_{\mathbf{r}}$ is a product, then $\mathbf{R}=(\mathbf{S T})_{\mathbf{r}}$, is also a product, where $\mathbf{r}^{\prime}=\mathrm{m} \circ\left(\phi_{S} * \phi_{T}\right)([8], 1.2,1.3,1.5)$.

1.3 If $\mathbf{T}=\left(\mathrm{T}, \eta_{\mathrm{T}}, \mu_{\mathrm{T}}\right)$ and $\mathbf{S}=\left(\mathrm{S}, \eta_{\mathrm{s}}, \mu_{\mathrm{S}}\right)$ are triples in $\mathbf{A}$, a distributive law of $\mathbf{T}$ over $\mathbf{S}$ is a natural transformation $\tau$ : TS $\Rightarrow$ ST which verifies:

D.L. 1) $\tau a\left(\eta_{\mathrm{T} * \mathrm{~S}}\right)=\mathrm{S} * \eta_{\mathrm{T}}$

D.L. 2) $\tau \circ\left(T * \eta_{S}\right)=\eta_{S *}$

D.L. 3) $\left(S \star \mu_{\mathrm{T}}\right) \circ(\tau \star T) \circ(\mathrm{T} \star \tau)=\tau \circ\left(\mu_{\mathrm{T}} \star S\right)$ ([2], 1).

D.L. 4) $\left(\mu_{S} * T\right) \circ(S * \tau) \circ(\tau * S)=\tau \circ\left(T * \mu_{S}\right)$

1.4 A distributive law $\tau$ of $\mathbf{T}$ over $\mathbf{S}$ makes a product $\mathbf{R}=(\mathbf{T S})_{\mathrm{r}}$ with $\mathbf{r}=$ $\tau, \mathbf{R}=\left(\mathrm{ST}, \eta_{\mathrm{S}} \star \eta_{\mathrm{T}},\left(\mu_{\mathrm{S}} \star \mu_{\mathrm{T}}\right) \circ(\mathrm{S} * \tau \star T)\right.$ and $\mathrm{r}^{\prime}=1_{\mathrm{ST}}$ ("half unitary law") $([8], 2.2)$. 
1.5 Conversely if $\mathbf{R}=(\mathrm{TS})_{\mathrm{r}}$ is a product with $\mathrm{R}=\mathrm{ST}$ and verifies the half unitary law, $r^{\prime}=1_{S T}$, then $r$ is a distributive law of $\mathbf{T}$ over $\mathbf{S}$ ([8], 2.3).

1.6 Taking one of the examples given in [2], we obtain a product (TS), in which $r$ is not a distributive law. In fact, if $T$ and $S$ are graduated rings, $R=S \otimes T$ is a ring with the product operation:

$$
\left(s_{1} \otimes t_{1}\right)\left(s_{2} \otimes t_{2}\right)=(-1)^{\partial s_{1} \partial t_{2}} s_{1} s_{2} \otimes t_{1} t_{2}
$$

( $\partial$ indicates the degree), being $1 \otimes 1$ the unity element. Moreover, the maps

are homomorphisms of rings.

$$
\begin{aligned}
& \phi_{\mathrm{T}}: \mathrm{T} \rightarrow \mathrm{T} \otimes \mathrm{S}, \phi_{\mathrm{T}}(\mathrm{t})=1 \otimes \mathrm{t} \\
& \phi_{\mathrm{S}}: \mathrm{S} \rightarrow \mathrm{S} \otimes \mathrm{T}, \phi_{\mathrm{S}}(\mathrm{s})=\mathrm{s} \otimes 1
\end{aligned}
$$

The rings $T, S$ and $R$ give the triples $T=\left(-\otimes T, \eta_{T}, \mu_{T}\right), S=(-\otimes S$, $\left.\eta_{S}, \mu_{S}\right)$ and $\mathbf{R}=\left(-\otimes R, \eta_{R}, \mu_{R}\right)$ in the category $\mathbf{A}$ of abelians groups (the natural transformations $\eta$ and $\mu$ are the ones induced by the unities and the multiplications of the rings). The homomorphisms $\phi_{\mathrm{T}}$ and $\phi_{\mathrm{S}}$ induce morphisms of triples

$$
\phi_{\mathrm{T}}: \mathbf{T} \Rightarrow \mathbf{R} \text { and } \phi_{\mathrm{S}}: \mathbf{S} \Rightarrow \mathbf{R}
$$

$\mathbf{R}=$ (TS) $)_{\mathrm{r}}$, being $\mathrm{r}=\mu_{\mathrm{R}}{ }^{\circ}\left(\phi_{\mathrm{T}} \star \phi_{\mathrm{S}}\right)(1.2)$. However, in this case $\mathrm{r}$ is not a distributive law, because the half unitary law is not verified, that is, $r^{\prime} \neq 1$.

\section{TENSOR PRODUCT OF TRIPLES}

2.1 Let $\mathrm{n} \in$ Set and $(-)^{\mathrm{n}}$ : Set $\rightarrow$ Set be the functor $\operatorname{Hom}(\mathrm{n},-)$. If $\mathbf{T}=\left(\mathrm{T}, \eta_{\mathrm{T}}, \mu_{\mathrm{T}}\right)$ is a triple in Set, a $n$-ary operation over $\mathrm{T}$ is a natural transformation $(-)^{n} \Rightarrow T$.

If $(X, \xi)$ is a $T$-algebra, each n-ary operation $g$ over $\mathbf{T}$ induces an operation, $\xi^{g}=\xi \circ(g X): X^{n} \rightarrow X$, over the set $X$ 


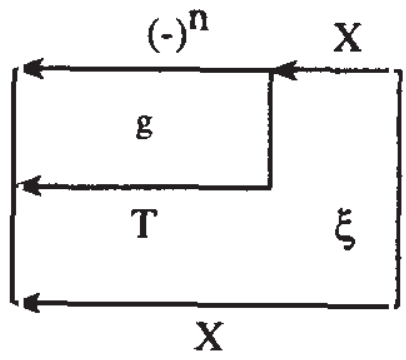

Moreover, a T-morphism $\mathrm{f}:(\mathrm{X}, \xi) \rightarrow(\mathrm{Y}, \boldsymbol{\theta})$ is a map $\mathrm{f:} \mathrm{X} \rightarrow \mathrm{Y}$ which is a morphism in the classic sense, commuting with each operation, that is, for each $\mathrm{g}:(-)^{\mathrm{n}} \Rightarrow \mathrm{T}$

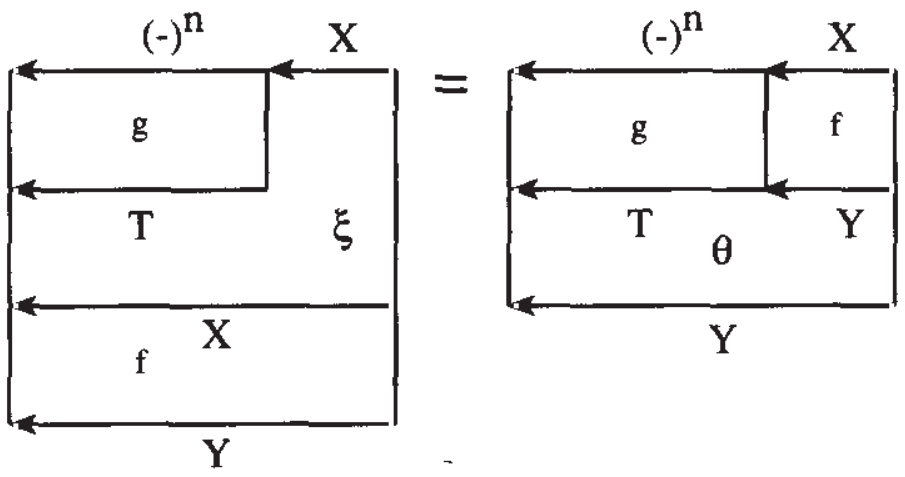

i.e.: $f \circ \xi^{g}=f \circ \xi \circ(g X)=\theta \circ(g * f)=\theta \circ(g Y) \circ f^{n}=\theta^{*} \circ f^{n}$ $([3], 1)$.

2.2 If $\mathbf{T}$ and $\mathbf{S}$ are triples in Set, a $\mathbf{S}$-T-bialgebra is a 3-triple $(\mathrm{X}, \sigma, \xi)$, with $(X, \sigma)$ and $S$-algebra and $(X, \xi)$ a $T$-algebra such that for all $n, m \in$ ISet, $\mathrm{g}:(-)^{\mathrm{n}} \Rightarrow \mathrm{T}$ and $\mathrm{h}:(-)^{\mathrm{m}} \Rightarrow \mathrm{S}$ the following holds true: 


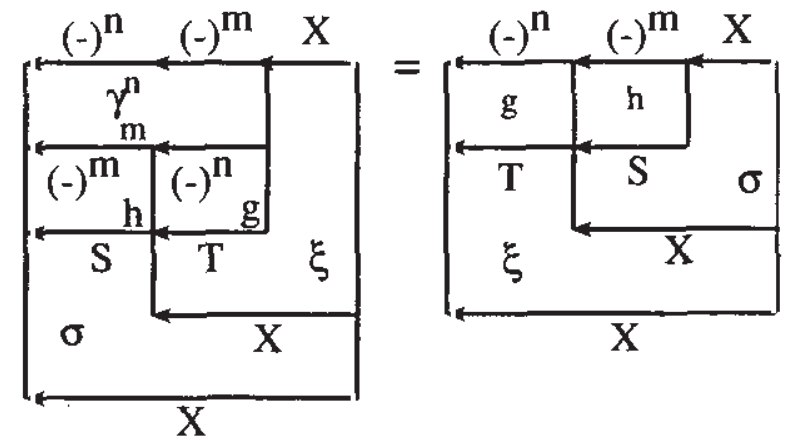

i.e.: $\sigma \circ\left(S_{\star} \xi\right) \circ\left(\left(h_{\star} g\right) X\right) \circ\left(\gamma_{m}^{n} X\right)=\xi \circ\left(T_{\star} \sigma\right) \circ\left(\left(g g_{\star} h\right) X\right)$, where

$$
\gamma_{m}^{n}:(-)^{n}(-)^{m} \Rightarrow(-)^{m}(-)^{n}
$$

is the canonical isomorphism.

This is equivalent to, for every $\mathrm{g:}(-)^{\mathrm{n}} \Rightarrow \mathrm{T}, \xi^{\mathrm{g}}$ is an S-morphism, or equivalently, for every $h:(-)^{m} \Rightarrow S, \sigma^{h}$ is a T-morphism $([3], 1)$.

This defines the category Set ${ }^{[\mathbf{S}, \mathbf{T}]}$ of S-T-bialgebras as a full subcategory of the category Set ${ }^{(\mathrm{S}, \mathrm{T})}$ whose objects are triples $(X, \sigma, \xi)$ with $(X, \sigma)$ an $\mathbf{S}$-algebra and $(X, \xi)$ a $\mathbf{T}$-algebra, and whose morphisms $\mathrm{f:}(\mathrm{X}, \sigma, \xi) \rightarrow(\mathrm{Y}, \tau, \theta)$ are maps $\mathrm{f}: \mathrm{X} \rightarrow \mathrm{Y}$, being $\mathrm{f}$ an $\mathrm{S}$-morphism and T-morphism.

If the forgetful functor $\mathrm{U}^{[\mathrm{S}, \mathrm{T}]}:$ Set ${ }^{[\mathrm{S}, \mathrm{T}]} \rightarrow$ Set is tripleable, it makes a triple $\mathbf{S} \otimes \mathbf{T}$ that is called tensor product (symmetrically, $\mathbf{T} \otimes \mathbf{S}$ ) ([3], [10], [11], [12]). question.

The existence of tensor product of triples is, in general, an open

\section{MULTILINEAR PRODUCTS}

3.1 Let $\mathbf{T}=\left(T, \eta_{T}, \mu_{T}\right)$ and $\mathbf{S}=\left(T, \eta_{\mathrm{S}}, \mu_{\mathrm{S}}\right)$ be triples in Set and $\mathbf{R}=$ (TS) $)_{\mathrm{r}}$ a product, $\mathbf{R}=\left(\mathrm{R}, \mathrm{r} \circ\left(\eta_{\mathrm{T} *} \eta_{\mathrm{S}}\right), \mathrm{m}\right)$. 
We will say that $\mathbf{R}$ is a multilinear product if for whatever $\mathrm{g}:(-)^{\mathrm{n}} \Rightarrow$ $\mathrm{T}$ and $\mathrm{h}:(-)^{\mathrm{m}} \Rightarrow \mathrm{S}$ it are verified
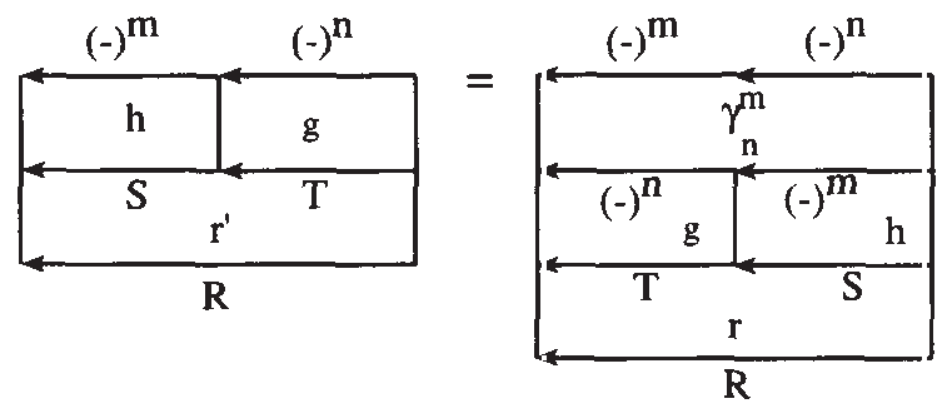

i.e.: $r^{\prime} \circ\left(h_{\star} g\right)=r \circ\left(g_{\star} h\right) \circ \gamma_{h}^{\prime \prime \prime}$.

3.2 If $\mathbf{R}=(\mathbf{T S})_{\mathbf{r}}$ is a product, the morphisms of triples $\phi_{S}: \mathbf{S} \Rightarrow \mathbf{R}$ and $\phi_{\mathrm{T}}: \mathbf{T} \Rightarrow \mathbf{R}(1.2)$ give functors (change of triple) $\mathrm{Set}^{\phi_{2}}: \mathrm{Set}^{\mathrm{R}} \rightarrow \mathrm{Set}^{\mathrm{S}}$ and Set $^{\text {thr }}: \operatorname{Set}^{\mathbf{R}} \rightarrow$ Set $^{\mathrm{T}}$, respectively, that commute with the forgetful functors to Set. As a result, each $\mathbf{R}$-algebra $(X, \tau)$ gives an $S$-algebra $\left(X, \sigma_{\tau}\right)=$ $\left(X, \tau \circ\left(\phi_{S} X\right)\right)$ and a $T$-algebra $\left(X, \xi_{\mathfrak{r}}\right)=\left(X, \tau \circ\left(\phi_{T} X\right)\right)$. Moreover, it is verified

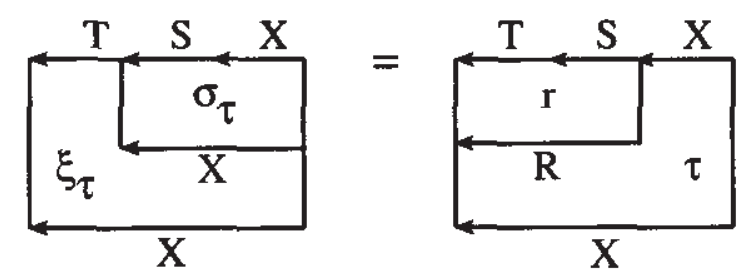

i.e.: $\xi_{\tau} \circ\left(T_{\star} \sigma_{\tau}\right)=\tau \star(r X)$, and
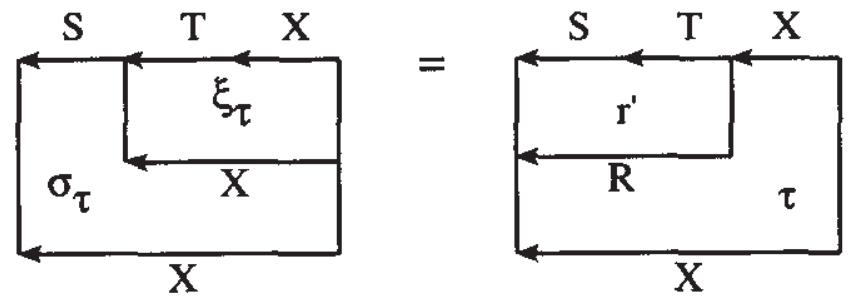

i.e.: $\sigma_{\tau} \circ\left(S_{\star} \xi_{\tau}\right)=\tau \circ\left(r^{\prime} X\right)[12]$, proposition 2.9, page 210). 
We will say that an $\mathbf{R}$-algebra $(X, \tau)$ is a bialgebra if the $\mathbf{S}$-algebra $\left(X, \sigma_{\tau}\right)$ and the $\mathbf{T}$-algebra $\left(X, \xi_{\tau}\right)$ make an $\mathbf{S}-\mathbf{T}$-bialgebra $\left(X, \sigma_{v}, \xi_{\tau}\right)$, i.e.:

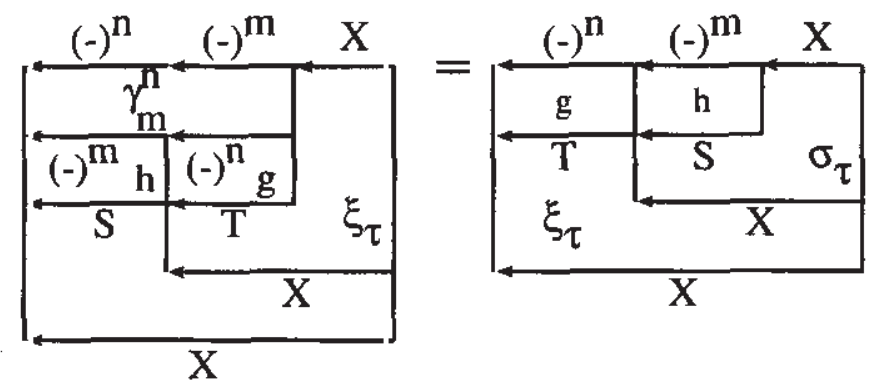

i.e.: $\sigma_{\tau} \circ\left(S_{\star} \xi_{\tau}\right) \circ\left(\left(h_{\star} g\right) X\right) \circ\left(\gamma_{m}^{n} X\right)=\xi_{\tau} \circ\left(T_{\star} \sigma_{\imath}\right) \circ\left(\left(g_{\star} h\right) X\right)$, for all operations $\mathrm{g}:(-)^{\mathrm{n}} \Rightarrow \mathrm{T}$ and $\mathrm{h}:(-)^{\mathrm{m}} \Rightarrow \mathrm{S}$.

It is immediately proved that the last equality is equivalent to:

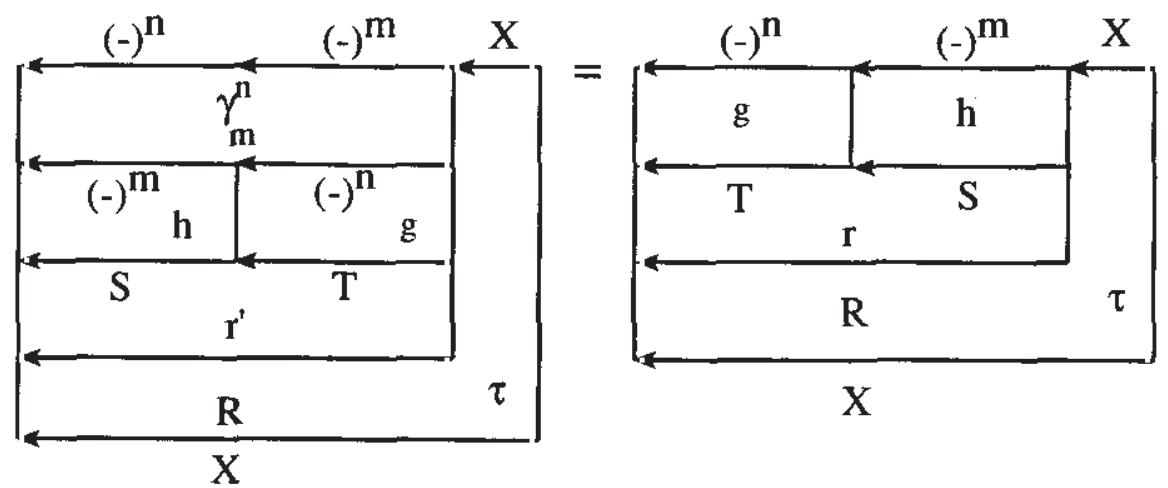

i.e.: $\tau \circ\left(r^{\prime} X\right) \circ\left(\left(h_{\star} g\right) X\right) \circ\left(\gamma_{m}^{n} X\right)=\tau \circ(r X) \circ\left(\left(g_{\star} h\right) X\right)$.

3.3 From former definitions we can conclude, trivially, that for a multilinear product $\mathbf{R}$, every $\mathbf{R}$-algebra is a bialgebra. Its reciprocal result is also true. If $\mathbf{R}$ is a product and every $\mathbf{R}$-algebra is a bialgebra, then $\mathbf{R}$ is multilinear as a consequence of the following result:

If $\mathbf{T}$ is a triple in Set and $\alpha, \beta:(-)^{k} \Rightarrow \mathrm{T}$ are k-ary operations over $\mathbf{T}$, then $\alpha=\beta$ if and only if $\tau^{\alpha}=\tau^{\beta}$ for every $\mathbf{T}$-algebra $(X, \tau)$ ([3], lemma 2.5 , page 145 ). 
To obtain our result, it is enough to take

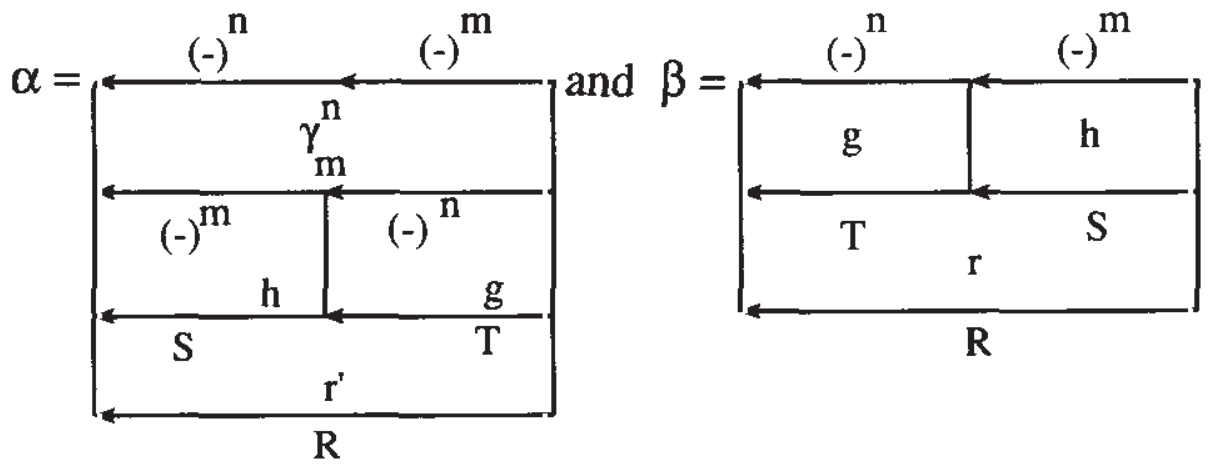

i.e.: $\alpha=r^{\prime} \circ\left(h_{\star} g\right) \circ \gamma_{m}^{n}$ and $\beta=r \circ\left(g_{\star} h\right)$.

\section{THE TENSOR PRODUCT AS MULTILINEAR PRODUCT}

4.1 Let $T=\left(T, \eta_{T}, \mu_{T}\right)$ and $S=\left(T, \eta_{\mathrm{S}}, \mu_{\mathrm{S}}\right)$ be triples in Set. Let us suppose that the tensor product $\mathbf{S} \otimes \mathbf{T}=\mathbf{R}=\left(\mathrm{R}, \eta_{\mathbf{R}}, \mathrm{m}\right)$ exist, i.e., the following diagram

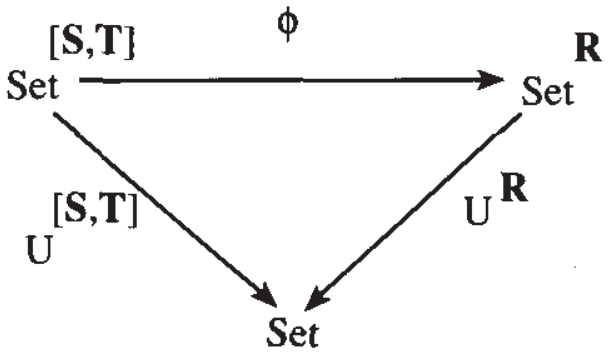

is commutative, $\phi$ being an isomorphism.

For the inclusion functor i: $\mathrm{Set}^{[\mathbf{S}, \mathbf{T}]} \rightarrow \mathrm{Set}^{\mathrm{s}}$,

$$
\left.\mathrm{i}\left(\mathrm{f}:(\mathrm{X}, \varepsilon, \delta) \rightarrow\left(\mathrm{X}^{\prime}, \varepsilon^{\prime}, \delta^{\prime}\right)\right)=\mathrm{f:}(\mathrm{X}, \varepsilon) \rightarrow\left(\mathrm{X}^{\prime}, \varepsilon^{\prime}\right)\right)
$$

the following diagram of functors is commutative: 


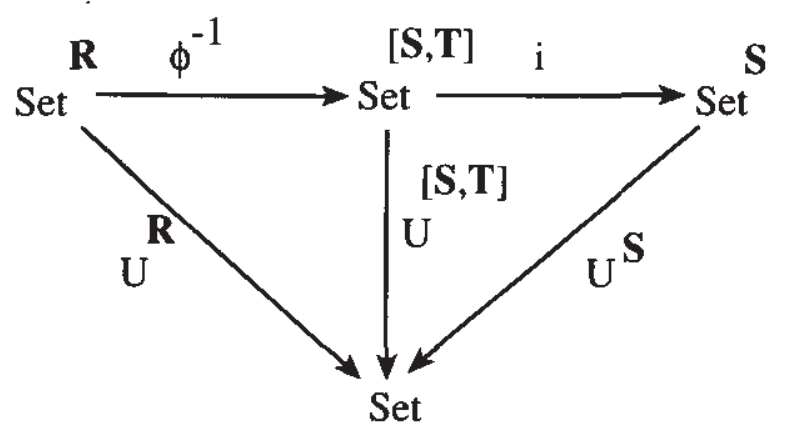

Since $i \circ \phi^{-1}$ commutes with the forgetful functors, a natural transformation exists $\sigma: S R \Rightarrow R$ such that $\phi_{S}=\sigma \circ\left(S_{\star} \eta_{R}\right): S \Rightarrow R$ is a morphism of triples ( $\mathrm{i} \circ \phi^{-1}=\mathrm{Set}^{\phi_{\mathrm{s}}}$ is the change of triple functor). For $X \in \mid$ Set $\mid$, and $(\mathrm{RX}, \mathrm{mX})$ being the free $R$-algebra over $\mathrm{X}$, $\left(\mathrm{i} \circ \phi^{-1}\right)(\mathrm{RX}, \mathrm{mX})=(\mathrm{RX}, \sigma \mathrm{X})$. For any $\mathrm{R}$-algebra $(\mathrm{X}, \tau)$, $\left(i \circ \phi^{-1}\right)(X, \tau)=\left(X, \tau \circ\left(\phi_{S} X\right)\right)$.

In the same way, for the triple $T$, a natural transformation exists $\xi$ : TR $\Rightarrow R$, such that $\phi_{T}=\xi \circ\left(T_{\star} \eta_{R}\right): T \Rightarrow R$ is a morphism of triples.

If $(X, \tau)$ is an $R$-algebra, $\phi^{-1}(X, \tau)=\left(X, \tau \circ\left(\phi_{\mathrm{S}} X\right), \tau \circ\left(\phi_{\mathrm{T}} X\right)\right)$. In particular, $\phi^{-1}(\mathrm{RX}, \mathrm{mX})=(\mathrm{RX}, \sigma \mathrm{X}, \xi \mathrm{X})$.

4.2 From all this and from (1.2) it follows that $R$ is a product, $R=(T S)_{r}$ with $\mathrm{r}=\mathrm{m} \circ\left(\phi_{\mathrm{T} *} \phi_{\mathrm{S}}\right)=\xi \circ\left(\mathrm{T}_{\star} \sigma\right) \circ\left(\mathrm{TS}_{*} \eta_{\mathrm{R}}\right)$ (this last equality is true since $\mathrm{mX}:\left(\mathrm{RRX}, \sigma \mathrm{RX}, \xi_{\mathrm{RX}}\right) \rightarrow(\mathrm{RX}, \sigma \mathrm{X}, \xi \mathrm{X})$ is a morphism of $\mathrm{S}$ algebras and of $\mathrm{T}$-algebras).

If $(X, \tau)$ is an $R$-algebra, $\phi^{-1}(X, \tau)=\left(X, \tau \circ\left(\phi_{S} X\right), \tau \circ\left(\phi_{T} X\right)\right)=(X$, $\left.\sigma_{\tau}, \xi_{\tau}\right)$ is an S-T-algebra and, by (3.2) and (3.3), $\mathbf{R}$ is a multilinear product. 


\section{THE CATEGORY OF MULTILINEAR PRODUCTS}

5.1 Let $T$ and $S$ be triples in Set. Taking as objects the multilinear products $\mathbf{R}=(\mathbf{T S})_{\mathrm{r}}$ and as morphisms, $\alpha: \mathbf{R}=(\mathbf{T S})_{\mathrm{r}} \rightarrow \mathbf{R}^{\prime}=(\mathbf{T S})_{\mathrm{r}^{\prime}}$, those morphisms of triples $\alpha: \mathbf{R} \rightarrow \mathbf{R}^{\prime}$ that make the diagram commutative

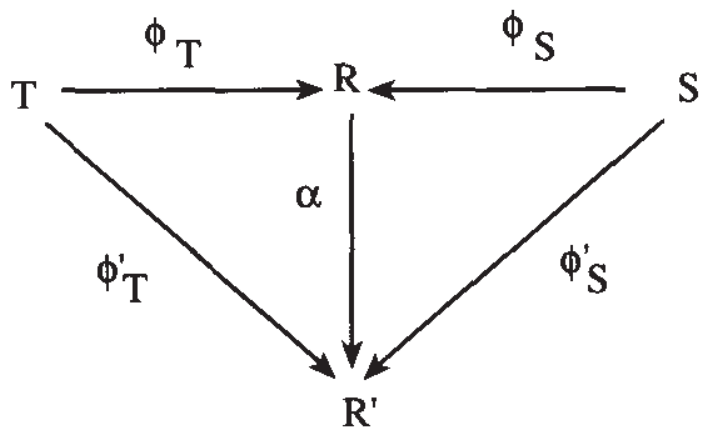

we obtain the category of multilinear products of $\mathbf{T}$ and $\mathbf{S}$.

5.2 In the category of multilinear products of $T$ and $S, S \otimes T$ is an initial object. Let us write $S \otimes T=R=\left(R, \eta_{R}, m\right)=(T S)_{r}$.

Let $\mathbf{R}^{\prime}=\left(\mathbf{R}^{\prime}, \eta_{\mathbf{R}^{\prime}}, \mathrm{m}^{\prime}\right)=(\mathbf{T S})_{\mathbf{r}^{\prime}}$, be an arbitrary multilinear product and $\phi^{\prime}{ }_{\mathrm{S}}: \mathbf{S} \Rightarrow \mathbf{R}$ ', $\phi_{\mathrm{T}}^{\prime}: \mathbf{T} \Rightarrow \mathbf{R}$ ' the corresponding morphisms of triples. If $\left(X, \tau^{\prime}\right)$ is an $\mathbf{R}^{\prime}$-algebra, $\left(X, \sigma_{\tau^{\prime}}, \xi_{\tau}\right)=\left(X, \tau^{\prime} \circ\left(\phi_{S}^{\prime} X\right), \tau^{\prime} \circ\left(\phi_{T}^{\prime} X\right)\right)$ is an S-T-bialgebra.

If $\mathrm{j}$ is the functor $\mathrm{j:} \operatorname{Set}^{\mathbf{R}^{\prime}} \rightarrow \operatorname{Set}^{[\mathrm{S}, \mathrm{T}]}$ such that $\mathrm{j}\left(\mathrm{X}, \tau^{\prime}\right)=\left(\mathrm{X}, \sigma_{\tau^{\prime}}, \xi_{\tau^{\prime}}\right)$, then the following diagram of functors is commutative: 


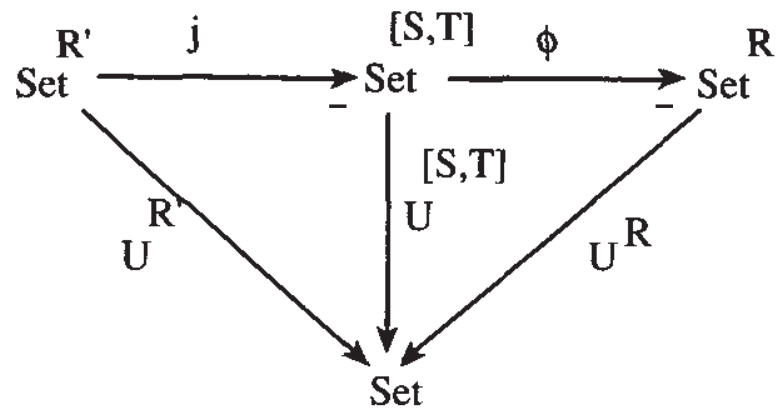

Thus, a natural transformation exists $\lambda: R^{\prime} \Rightarrow R^{\prime}$, so that $\alpha=\lambda \circ\left(\mathbf{R}_{\star} \eta_{\mathbf{R}}\right): \mathbf{R} \Rightarrow \mathbf{R}^{\prime}$ is a morphism of triples, being $\phi \circ j=\operatorname{Set}^{\alpha}$ the change of triple functor. Moreover, $(\phi \circ j)\left(X, \tau^{\prime}\right)=\left(X, \tau^{\prime} \circ(\alpha, X)\right)$ and, in particular, $(\phi \circ j)\left(R^{\prime} X, m^{\prime} X\right)=\left(R^{\prime} X, \lambda X\right)=\left(R^{\prime} X,\left(m^{\prime} X\right) \circ\right.$ $\left.\left(\alpha R^{\prime} X\right)\right)$. Then,

$\left(R^{\prime} X,\left(m^{\prime} X\right) \circ\left(\phi_{S}^{\prime} R^{\prime} X\right),\left(m^{\prime} X\right) \circ\left(\phi_{T}^{\prime} R^{\prime} X\right)\right)=\phi^{-1}\left((\phi \circ j)\left(R^{\prime} X, m^{\prime} X\right)=\right.$ $=\phi^{-1}\left(R^{\prime} X,\left(m^{\prime} X\right) \circ\left(\alpha R^{\prime} X\right)\right)=\left(R^{\prime} X,\left(m^{\prime} X\right) \circ\left(\alpha R^{\prime} X\right) \circ\left(\phi_{S} R^{\prime} X\right),\left(m^{\prime} X\right)\right.$ $\left.\circ\left(\alpha R^{\prime} X\right) \circ\left(\phi_{T} R^{\prime} X\right)\right)$ from which we can obtain

$$
\begin{aligned}
& m^{\prime} \circ\left(\phi_{S_{\star}}^{\prime} R^{\prime}\right)=m^{\prime} \circ\left(\left(\alpha \circ \phi_{S}\right)_{\star} R^{\prime}\right) \Leftrightarrow \phi_{S}^{\prime}=\alpha \circ \phi_{S} \\
& m^{\prime} \circ\left(\phi^{\prime}{ }_{T \star} R^{\prime}\right)=m^{\prime} \circ\left(\left(\alpha \circ \phi_{T}\right)_{\star} R^{\prime}\right) \Leftrightarrow \phi_{T}^{\prime}=\alpha \circ \phi_{T}
\end{aligned}
$$

that is, there is a morphism in the category of multilinear products $\alpha: \mathbf{R} \rightarrow \mathbf{R}^{\prime}$.

5.3 Let us see the uniqueness of $\alpha$. Let $\beta: \mathbf{R} \rightarrow \mathbf{R}$ ' be a morphism of triples such that $\beta \circ \phi_{\mathrm{T}}=\phi_{\mathrm{T}}^{\prime}$ and $\beta \circ \phi_{\mathrm{S}}=\phi_{\mathrm{S}}{ }_{\mathrm{S}}$. $\mathrm{Set}^{\beta}$ : $\mathrm{Set}^{\mathbf{R}^{\prime}} \rightarrow \mathrm{Set}^{\mathbf{R}}$ is the change of triple functor corresponding to $\beta$ (note that $\phi \circ j=\operatorname{Set}^{\alpha}$ ). Then

$$
\alpha=\beta \Leftrightarrow \operatorname{Set}^{\alpha}=\operatorname{Set}^{\beta} \Leftrightarrow \operatorname{Set}^{\alpha}\left(\mathrm{R}^{\prime} \mathrm{X}, \mathrm{m}^{\prime} \mathrm{X}\right)=\operatorname{Set}^{\beta}\left(\mathrm{R}^{\prime} \mathrm{X}, \mathrm{m}^{\prime} \mathrm{X}\right) \text { for }
$$
every free $\mathbf{R}^{\prime}$-algebra ( $\mathrm{R}^{\prime} \mathrm{X}, \mathrm{m}^{\prime} \mathrm{X}$ ) ([12], prop. 2.9, page 210). Also 


$$
\begin{aligned}
& \beta \circ \phi_{T}=\phi_{T}^{\prime}=\alpha \circ \phi_{T} \Leftrightarrow m^{\prime} \circ\left(\beta_{\star} R^{\prime}\right) \circ\left(\phi_{T^{\star}} R^{\prime}\right)=m^{\prime} \circ\left(\alpha_{*} R^{\prime}\right) \circ\left(\phi_{T^{\star}} R^{\prime}\right) \\
& \beta \circ \phi_{S}=\phi_{S}^{\prime}=\alpha \circ \phi_{S} \Leftrightarrow m^{\prime} \circ\left(\beta_{\star} R^{\prime}\right) \circ\left(\phi_{S^{\star}} R^{\prime}\right)=m^{\prime} \circ\left(\alpha_{\star} R^{\prime}\right) \circ\left(\phi_{S \star} R^{\prime}\right)
\end{aligned}
$$

But, j(R'X, m'X) $=\left(R^{\prime} X,\left(m^{\prime} X\right) \circ\left(\alpha R^{\prime} X\right) \circ\left(\phi_{s} R^{\prime} X\right),\left(m^{\prime} X\right) \circ\left(\alpha R^{\prime} X\right)\right.$ $\left.\circ\left(\phi_{T} R^{\prime} X\right)\right)=\phi^{-1} \operatorname{Set}^{\beta}\left(R^{\prime} X, m^{\prime} X\right)$, so $\operatorname{Set}^{\alpha}\left(R^{\prime} X, m^{\prime} X\right)=\operatorname{Set}^{\beta}\left(R^{\prime} X, m^{\prime} X\right)$,

\section{References}

[1] BARR, M. Coequalizers and free triples. Math. Z. 116 (1970), 307-322.

[2] BECK, J. Distributive laws. Lect. Notes in Math. 80. Springer (1969), 119-140.

[3] BUNGE, M. On the relationship between composite and tensor product triples. Journal of Pure and Applied Algebra 13 (1978), 139-156.

[4] CARUNCHO CASTRO, J.R. Teoría de triples. Alxebra 5. Dpto. Álgebra y Fundamentos. Santiago (1971).

[5] FREIRE NISTAL, J.L. Propiedades universales de triples de grado superior. Tesis. Alxebra 11, Dpto. Algebra y Fundamentos. Santiago (1971).

[6] FREYD, P. Algebra-valued functors in general and tensor products in particular. Colloq. Mathem. XIV (1966), 89-106.

[7] LOPEZ LOPEZ, M.A. Algebras de Hopf respecto a un cotriple. Tesis. Alxebra 17, Dpto. Álgebra y Fundamentos. Santiago (1976).

[8] LOPEZ LOPEZ, M.A. y VILLANUEVA NOVOA, E. Producto de triples. Rev. Mat. Hisp.-Amer. T. 38 (1978), 61-70.

[9] LOPEZ LOPEZ, M.A. y BARJA PEREZ, J.M. El triple $\operatorname{Hom}(M,-)$ en conjuntos. Alxebra 26. Dpto. Álgebra y Fundamentos. Santiago (1980), 51-61.

[10] MANES, E. A triple miscellany. Thesis. Wesleyan University (1967).

[11] MANES, E. A triple theoretic construction of compact algebras. Lect. Not. in Math. 80. Springer (1969), 91-118.

[12] MANES, E. Algebraic Theories. Springer (1976).

Departamento de Computación Universidad de La Coruña 15071 La Corứa SPAIN

Departamento de Álgebra Universidad de Santiago 15771 Santiago de Compostela SPAIN 